\title{
Synthesis of quinolino $\left[2^{\prime}, 3^{\prime}: 8,7\right] \operatorname{cyclooct}[b]$ indole
}

\author{
LEENA VAIRAVELU and K J RAJENDRA PRASAD* \\ Department of Chemistry, Bharathiar University, Coimbatore 641 046, India \\ e-mail: prasad_125@yahoo.com
}

MS received 21 November 2013; revised 17 February 2014; accepted 18 February 2014

\begin{abstract}
A rapid and efficient synthetic route for the synthesis of 7,8,9,10-tetrahydroquinolino $\left[2^{\prime}, 3^{\prime}: 8,7\right]$ cyclooct $[b]$ indole analogues has been developed by reaction of 1 -oxo-1,2,3,4,5,6-hexahydrocyclooct $[b]$ indole with anthranilic acid and 3-amino pyrazine acid under $\mathrm{POCl}_{3}$ condition and the synthesis of 7,8,9,10tetrahydroquinolino $\left[2^{\prime}, 3^{\prime}: 8,7\right]$ cyclooct $[b]$ indole-6-carboxylic acid has been designed by reaction of 1 -oxo$1,2,3,4,5,6$-hexahydrocyclooct $[b]$ indole with isatin in the presence of $\mathrm{NaOH}$ via Pfitzinger reaction. These methods are more satisfactory in terms of the yield and simple one-pot operation. Structures of the products thus obtained were confirmed by spectral studies.
\end{abstract}

Keywords. 7,8,9,10-Tetrahydroquinolino[ $\left[2^{\prime}, 3^{\prime}: 8,7\right]$ cyclooct $[b]$ indole; 1 -oxo-1,2,3,4,5,6-hexahydrocyclooct $[b]$ indole; Pfitzinger reaction.

\section{Introduction}

Among the nitrogen heterocycles, indole is an important structural components in alkaloids and many pharmaceutical agents. Indole exhibits a high degree of biological activities including antifungal, antibacterial, antitumour, anti-HIV and DNA interactions. Substituted indoles have been referred to as 'privileged structures' since they are capable of binding to many receptors with high affinity. ${ }^{1}$ The structural diversity and biological importance of indole have made them attractive targets for synthesis over many years. Cyclooct[b]indoles, a sub-class of the indoles, represent an important part of many naturally occurring alkaloids, such as macroline (1), ajmaline (2), macrocarpamine (3), villalstonine (4), $O$-acetyl macralstonine (5a) and macralstonine (5b) with highly interesting pharmacological properties (figure 1). The biological activities of cyclooct $[b]$ indoles cover wide spectrum which include antiamoebic, antiplasmodic, antiprotozol and antihypertensive activities. ${ }^{2-5}$ The diverse biological activities of macrolines validates the cyclooct $[b]$ indoles system as a promising scaffold for the generation of bioactive compounds (figure 1). The structure of cyclooct[b] indole core ${ }^{6}$ is represented in figure 2.

Quinoline or 1-aza-napthalene or benzo[b]pyridine is nitrogen-containing heterocyclic aromatic compound. It is a system present in many classes of biologically active compounds. A number of quinoline related

\footnotetext{
*For correspondence
}

compounds have been clinically used as antifungal, antibacterial, and antiprotozoic drugs ${ }^{7-10}$ as well as antituberculosis agents. ${ }^{11-13}$ Some quinoline analogues also showed antineoplastics activity. ${ }^{14}$ Recently, styrylquinoline derivatives have gained remarkable attention due to their activity as potential HIV integrase inhibitors. ${ }^{15-19}$ The pyrazine ring is a part of many polycyclic compounds of biological and industrial significance. Examples are quinoxalines, phenazines, bioluminescent natural products, pteridines, flavins, and their derivatives. Pyrazino[3,2,1- $j, k]$ carbazoles represent a new class of heterocyclic compounds possessing interesting pharmacological properties (figure 3). $3 H$-pyrazino[3,2,1- $j, k]$ carbazoles possess neuroleptic properties and hence are of pharmacological interest.

Inspired by the wide range of useful activities possessed by cyclooct $[b]$ indole, quinoline, and pyrazino pyrido derivatives and in continuation of our efforts in search of bioactive compounds, combination of cyclooct $[b]$ indole, quinoline and pyrazino pyrido structures may therefore lead to play a vital role in biological as well as pharmaceutical systems. Considering these facts, our strategy is to couple quinoline and pyrazino pyrido moiety with cyclooct $[b]$ indole nucleus to obtain a new class of compounds, the quinolino cyclooct[$[b]$ indole and pyrazino $\left[2^{\prime \prime}, 3^{\prime \prime}-\mathrm{e}^{\prime}\right]$ pyrido- $\left[2^{\prime}, 3^{\prime}: 8,7\right]$ cyclooct $[b]$ indole derivatives. In some cases, specific substitution patterns like quinolino cyclooct $[b]$ indole and pyrazino[ $\left[2^{\prime \prime}, 3^{\prime \prime}-\right.$ $\left.\mathrm{e}^{\prime}\right]$ pyrido- $\left[2^{\prime}, 3^{\prime}: 8,7\right]$ cyclooct $[b]$ indole are difficult to obtain by standard pyrazino pyrido forming or quinoline forming reactions, therefore, new methodologies 


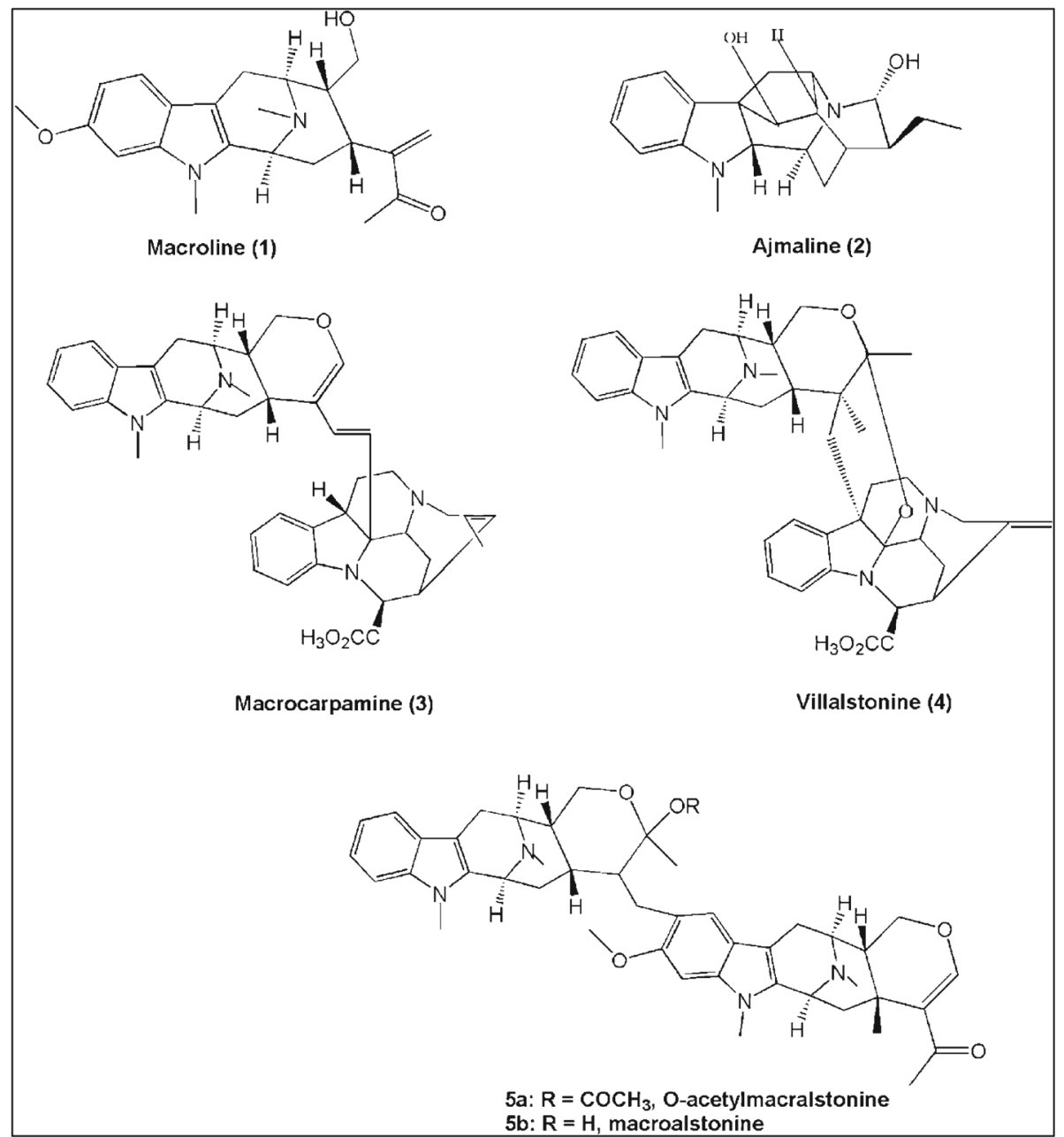

Figure 1. Structures of naturally occurring alkaloids having cyclooct $[b]$ indole core.

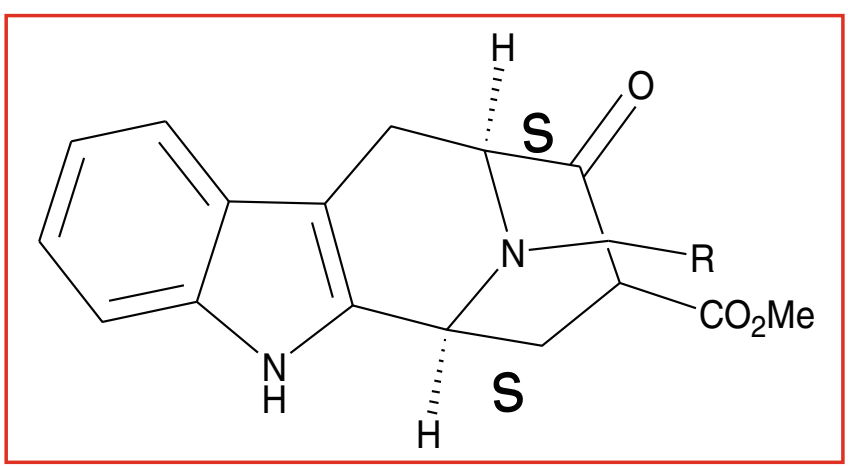

Figure 2. Structure of cyclooct $[b]$ indole core.

emerged. Prompted by all these observations, a simple strategy has been planned to synthesize a new class of cyclooct $[b]$ indole derivatives possessing quinoline and pyrazino pyrido moiety in their structure with more potent activity. This manuscript represents the construction of quinolino cyclooct $[b]$ indole and pyrazino $\left[2^{\prime \prime}, 3^{\prime \prime}-\mathrm{e}^{\prime}\right]$

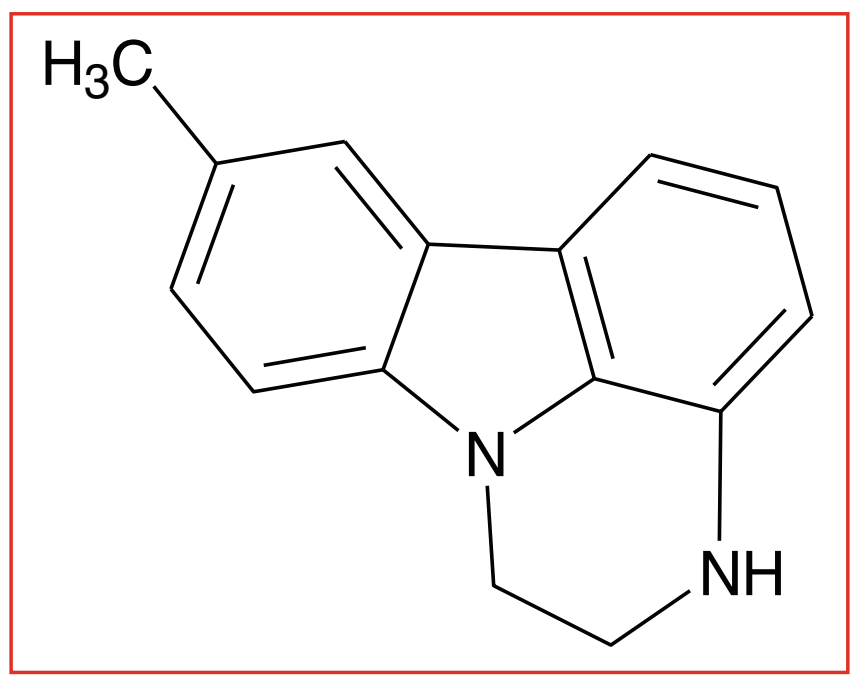

Figure 3. Structure of pyrazino $[3,2,1-j, k]$ carbazole.

pyrido- $\left[2^{\prime}, 3^{\prime}: 8,7\right]$ cyclooct $[b]$ indole derivatives, using 1 oxo-1,2,3,4,5,6-hexahydrocyclooct $[b]$ indole $\mathbf{6}$ as the potential precursors. 
The general synthetic methods shown in schemes 1, 2 and 3 are employed to prepare the quinolinocyclooct $[b]$ indole and pyrazino $\left[2^{\prime \prime}, 3^{\prime \prime}-\mathrm{e}^{\prime}\right]$ pyrido- $\left[2^{\prime}, 3^{\prime}\right.$ : $8,7]$ cyclooct $[b]$ indole derivatives 8a-d, 10a-d and 11a-d. The synthesis of quinolino cyclooct $[b]$ indole and pyrazino $\left[2^{\prime \prime}, 3^{\prime \prime}-\mathrm{e}^{\prime}\right]$ pyrido-[2',3':8,7]cyclooct[$\left.b\right]$ indole compounds were realized in $\mathrm{POCl}_{3}$ in a single process and quinolino cyclooct $[b]$ indole-6-carboxylic acid are prepared under Pfitzinger condition.

\section{Experimental}

\subsection{General}

Melting points (m.p.) were determined on Mettler FP 51 apparatus (Mettler Instruments, Switzerland) and are uncorrected. They are expressed in degree centigrade $\left({ }^{\circ} \mathrm{C}\right)$. A Nicolet Avatar Model FT-IR spectrophotometer is used to record the IR spectra $\left(4000-400 \mathrm{~cm}^{-1}\right)$.

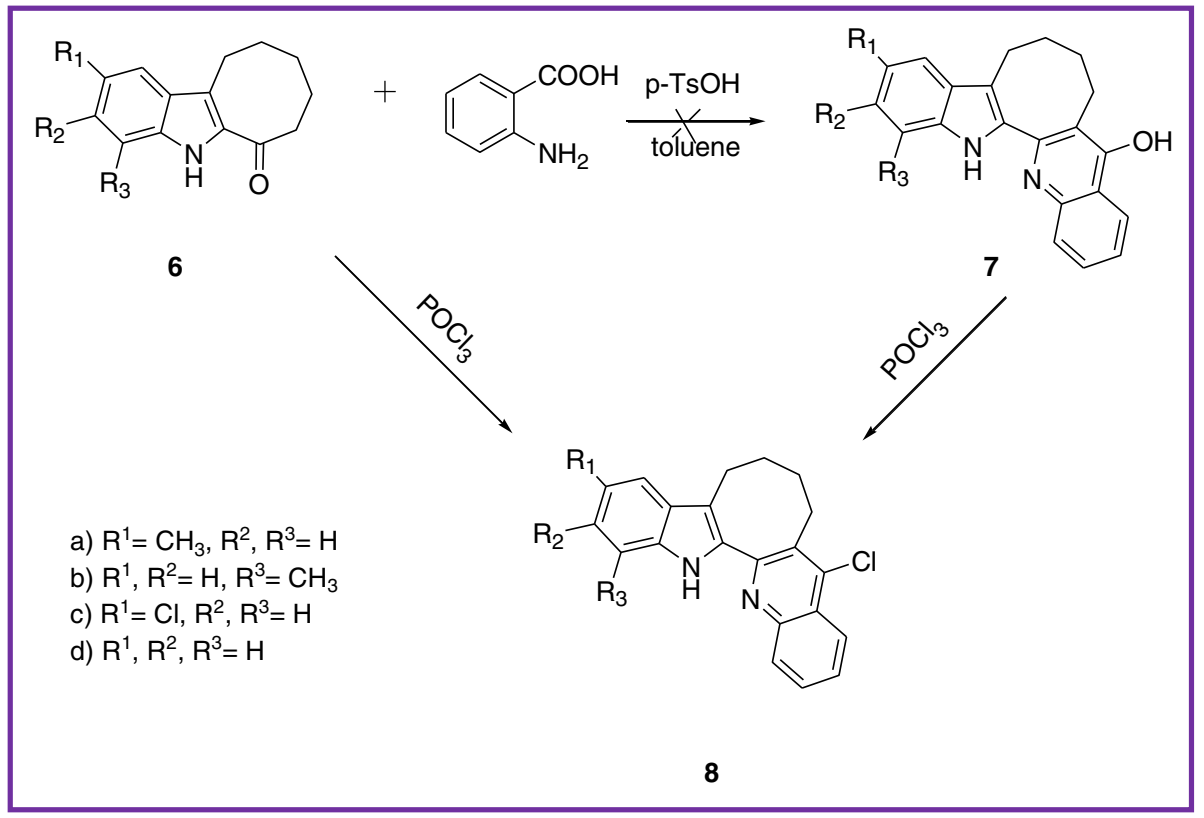

Scheme 1. $\mathrm{POCl}_{3}$ catalysed synthesis of 6-chloro-7,8,9,10-tetrahydroquinolino[2', $\left.3^{\prime}: 8,7\right]$ cyclooct $[b]$ indole.

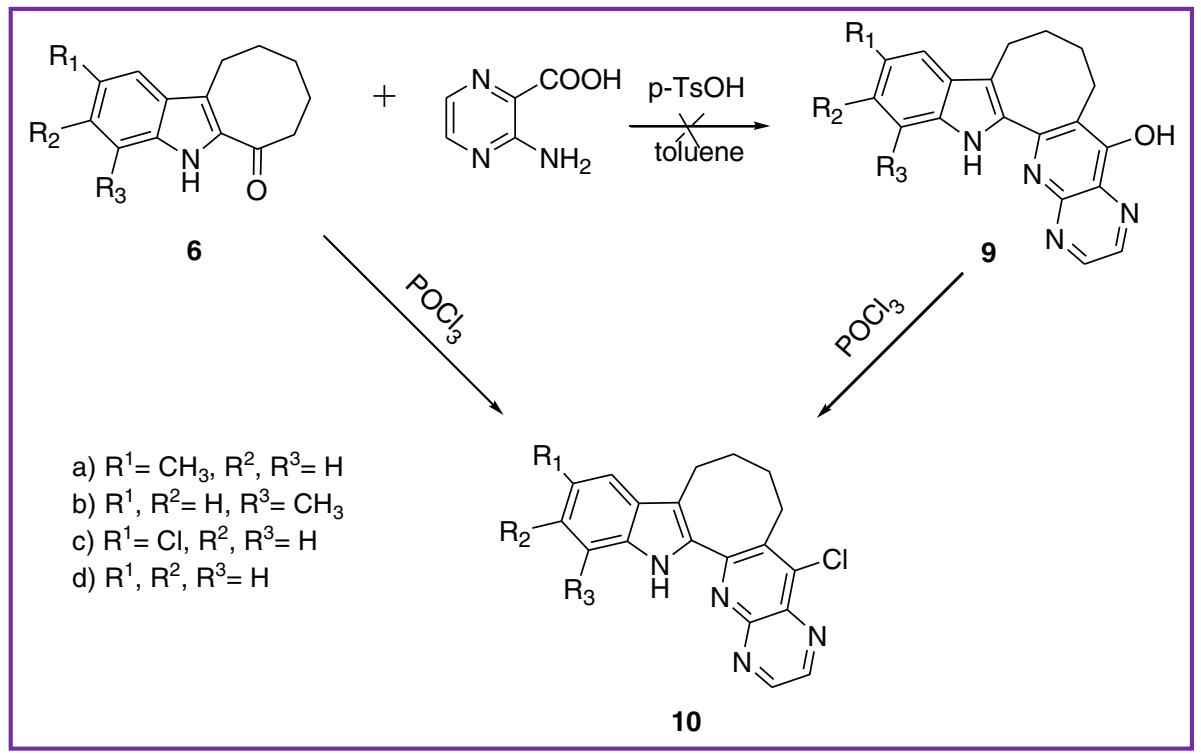

Scheme 2. $\mathrm{POCl}_{3}$ catalysed synthesis of 6-chloro-7,8,9,10-tetrahydro(pyrazino $\left[2^{\prime \prime}, 3^{\prime \prime}-\mathrm{e}^{\prime}\right]$ pyrido)- $\left[2^{\prime}, 3^{\prime}: 8,7\right]$ cyclooct $[b]$ indole. 


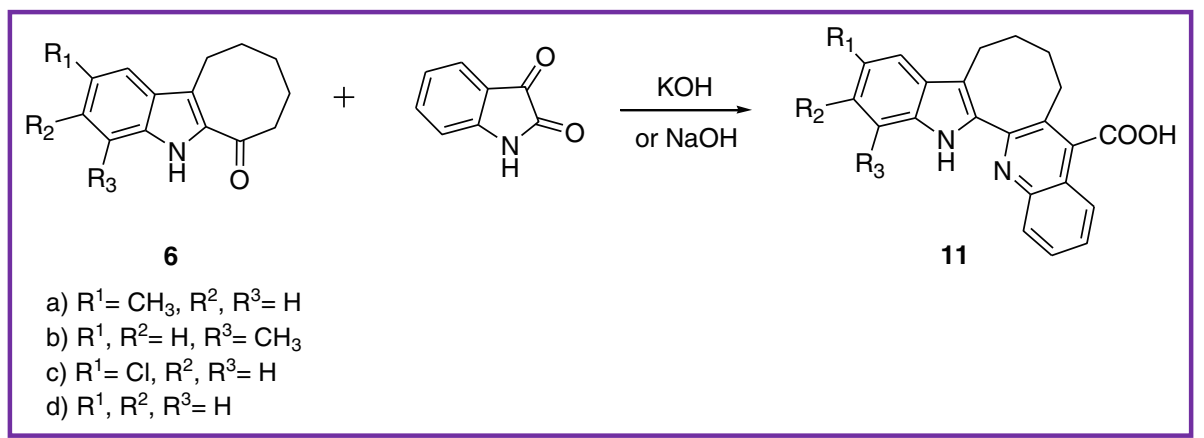

Scheme 3. Synthesis of 7,8,9,10-tetrahydroquinolino[2',3':8,7]cyclooct $[b]$ indole-6-carboxylic acid via Pfitzinger reaction.

${ }^{1} \mathrm{H}$ NMR and ${ }^{13} \mathrm{C}$ NMR spectra were recorded on Bruker AV $500\left(500 \mathrm{MHz}\left({ }^{1} \mathrm{H}\right)\right.$ and $\left.125 \mathrm{MHz}\left({ }^{13} \mathrm{C}\right)\right)$ spectrometer using tetramethylsilane (TMS) as an internal standard. The chemical shifts are expressed in parts per million (ppm). Mass spectra (MS), were recorded on Auto Spec EI+ shimadzu QP 2010 PLUS GC-MS mass spectrometer. Microanalyses were performed on a Vario EL III model CHNS analyzer (Vario, Germany) at the Department of Chemistry, Bharathiar University. Developing solvents were coated with silica Gel- G, petroleum ether and ethyl acetate.

\subsection{General procedure for the synthesis}

of 6-chloro-7,8,9,10-tetrahydroquinolino [2', $\left.3^{\prime}: 8,7\right]$ cyclooct[b]indole $(\mathbf{8 a}-\mathbf{d})$

A mixture of 1-oxo-1,2,3,4,5,6-hexahydrocyclooct[b] indole $(6,1 \mathrm{mmol})$, anthranilic acid $(1 \mathrm{mmol})$ in $20 \mathrm{~mL}$ of phosphorous oxychloride was refluxed at $140^{\circ} \mathrm{C}$ for $16 \mathrm{~h}$. The reaction was monitored by TLC. After the completion, the reaction mixture was poured into ice water with constant stirring and the $\mathrm{pH}$ was adjusted to 8 by adding $10 \% \mathrm{NaOH}$ solution. The precipitate formed was filtered off and dried. The crude product thus obtained was purified by column chromatography over silica gel using petroleum ether:ethyl acetate mixture (99:1) and recrystallised from ethanol to yield the corresponding product 6-chloro-7,8,9,10tetrahydroquinolino $\left[2^{\prime}, 3^{\prime}: 8,7\right]$ cyclooct $[b]$ indole 8 .

2.2a 6-Chloro-12-methyl-7,8,9,10-tetrahydroquinolino[2',3':8,7]cyclooct[b]indole $(8 \boldsymbol{a})$ : Pale yellow solid; yield: 56\%; M.p. $229-231^{\circ} \mathrm{C} \mathrm{IR}\left(\mathrm{KBr}, \mathrm{cm}^{-1}\right) v_{\max }$ : $3458(\mathrm{~N}-\mathrm{H}), 1572(\mathrm{C}=\mathrm{N}) .{ }^{1} \mathrm{H}$ NMR $\left(\mathrm{CDCl}_{3}\right) \delta: 9.00$ (b s, $\left.1 \mathrm{H}, \mathrm{N}_{15}-\mathrm{H}\right), 8.30-7.15\left(\mathrm{~m}, 7 \mathrm{H}, \mathrm{C}_{2}, \mathrm{C}_{3}, \mathrm{C}_{4}, \mathrm{C}_{5}\right.$, $\mathrm{C}_{11}, \mathrm{C}_{13}$ and $\left.\mathrm{C}_{14}-\mathrm{H}\right), 3.50-1.00\left(\mathrm{~m}, 8 \mathrm{H}, \mathrm{C}_{7}, \mathrm{C}_{8}, \mathrm{C}_{9}\right.$ and $\left.\mathrm{C}_{10}-\mathrm{CH}_{2}\right), 2.50\left(\mathrm{~s}, 3 \mathrm{H}, \mathrm{C}_{12}-\mathrm{CH}_{3}\right) ;{ }^{13} \mathrm{C}$ NMR $\left(\mathrm{CDCl}_{3}\right) \delta$ :
$156.8\left(\mathrm{C}_{1 \mathrm{a}}\right), 142.3\left(\mathrm{C}_{2 \mathrm{a}}\right), 137.8\left(\mathrm{C}_{6}\right), 132.3\left(\mathrm{C}_{3}\right), 130.4$ $\left(\mathrm{C}_{15 \mathrm{a}}\right), 130.2\left(\mathrm{C}_{1 \mathrm{~b}}\right), 129.5\left(\mathrm{C}_{7 \mathrm{a}}\right), 128.7\left(\mathrm{C}_{2}\right), 128.2$ $\left(\mathrm{C}_{4}\right), 127.3\left(\mathrm{C}_{11 \mathrm{a}}\right), 126.5\left(\mathrm{C}_{5}\right), 125.5\left(\mathrm{C}_{6 \mathrm{a}}\right), 124.4\left(\mathrm{C}_{12}\right)$, $123.8\left(\mathrm{C}_{11}\right), 123.5\left(\mathrm{C}_{1 \mathrm{~b}}\right), 119.4\left(\mathrm{C}_{13}\right), 111.5\left(\mathrm{C}_{14}\right), 28.5$ $\left(\mathrm{C}_{7}\right), 27.6\left(\mathrm{C}_{8}\right), 26.4\left(\mathrm{C}_{9}\right), 21.9\left(\mathrm{C}_{12}-\mathrm{CH}_{3}\right), 19.6\left(\mathrm{C}_{10}\right)$; MS : m/z (\%) $346\left(\mathrm{M}^{+} 100 \%\right), 348(\mathrm{M}+2,18 \%)$; Anal. calcd. for $\mathrm{C}_{22} \mathrm{H}_{19} \mathrm{~N}_{2} \mathrm{Cl}: \mathrm{C}, 76.18 ; \mathrm{H}, 5.52, \mathrm{~N}$, 8.08. Found: C, 76.20; H, 5.53 N, 8.10\%.

2.2b 6-Chloro-14-methyl-7,8,9,10-tetrahydroquinolino $\left[2^{\prime}, 3^{\prime}: 8,7\right]$ cyclooct [b]indole $(8 \boldsymbol{b})$ : Yellow solid; yield: $43 \%$; M.p. $225-227^{\circ} \mathrm{C}$, IR $\left(\mathrm{KBr}, \mathrm{cm}^{-1}\right) v_{\max }$ : $3288(\mathrm{~N}-\mathrm{H}), 1582(\mathrm{C}=\mathrm{N}) .{ }^{1} \mathrm{H} \mathrm{NMR}\left(\mathrm{CDCl}_{3}\right) \delta: 9.50(\mathrm{~b} \mathrm{~s}$, $\left.1 \mathrm{H}, \mathrm{N}_{15}-\mathrm{H}\right), 7.80-6.90\left(\mathrm{~m}, 7 \mathrm{H}, \mathrm{C}_{2}, \mathrm{C}_{3}, \mathrm{C}_{4}, \mathrm{C}_{5}, \mathrm{C}_{11}, \mathrm{C}_{12}\right.$ and $\left.\mathrm{C}_{13}-\mathrm{H}\right), 3.40-3.60\left(\mathrm{~m}, 4 \mathrm{H}, \mathrm{C}_{7}, \& \mathrm{C}_{10}-\mathrm{CH} 2\right), 2.50$ (s, $\left.3 \mathrm{H}, \mathrm{C}_{12}-\mathrm{CH}_{3}\right), 2.10-1.20\left(\mathrm{~m}, 4 \mathrm{H}, \mathrm{C}_{8} \& \mathrm{C}_{9}-\mathrm{CH}_{2}\right) ;{ }^{13} \mathrm{C}$ NMR $\left(\mathrm{CDCl}_{3}\right) \delta$ : $156.4\left(\mathrm{C}_{1 \mathrm{a}}\right), 142.3\left(\mathrm{C}_{2 \mathrm{a}}\right), 137.7\left(\mathrm{C}_{6}\right)$, $132.2\left(\mathrm{C}_{3}\right), 131.3\left(\mathrm{C}_{15 \mathrm{a}}\right), 130.3\left(\mathrm{C}_{1 \mathrm{~b}}\right), 130.0\left(\mathrm{C}_{7 \mathrm{a}}\right)$, $128.9\left(\mathrm{C}_{2}\right), 128.8\left(\mathrm{C}_{4}\right), 127.5\left(\mathrm{C}_{11 \mathrm{a}}\right), 126.7\left(\mathrm{C}_{5}\right), 125.4$ $\left(\mathrm{C}_{6 \mathrm{a}}\right), 124.6\left(\mathrm{C}_{12}\right), 123.7\left(\mathrm{C}_{11}\right), 123.6\left(\mathrm{C}_{11 \mathrm{~b}}\right), 118.8$ $\left(\mathrm{C}_{13}\right), 111.4\left(\mathrm{C}_{14}\right), 28.2\left(\mathrm{C}_{7}\right), 27.3\left(\mathrm{C}_{8}\right), 26.4\left(\mathrm{C}_{9}\right)$, $21.4\left(\mathrm{C}_{14}-\mathrm{CH}_{3}\right), 19.4\left(\mathrm{C}_{10}\right)$; $\mathrm{MS}: \mathrm{m} / \mathrm{z}(\%) 346\left(\mathrm{M}^{+}\right.$ $100 \%), 348(\mathrm{M}+2,18 \%)$; Anal. calcd.for $\mathrm{C}_{22} \mathrm{H}_{19} \mathrm{~N}_{2} \mathrm{Cl}$ : C, 76.18; H, 5.52, N, 8.08. Found: C, 76.20; H, 5.53 N, $8.10 \%$.

2.2c 6,12-Dichloro-7,8,9,10-tetrahydroquinolino$\left[2^{\prime}, 3^{\prime}: 8,7\right]$ cyclooct [b]indole $(8 c)$ : Orange yellow solid; yield: $42 \%$; M.p. $241-242^{\circ} \mathrm{C}$, IR $(\mathrm{KBr}$, $\left.\mathrm{cm}^{-1}\right) v_{\max }: 3297(\mathrm{~N}-\mathrm{H}), 1588(\mathrm{C}=\mathrm{N}) .{ }^{1} \mathrm{H}$ NMR $\left(\mathrm{CDCl}_{3}\right) \delta: 9.20\left(\mathrm{~b} \mathrm{~s}, 1 \mathrm{H}, \mathrm{N}_{15}-\mathrm{H}\right), 7.70-7.10(\mathrm{~m}, 7 \mathrm{H}$, $\mathrm{C}_{2}, \mathrm{C}_{3}, \mathrm{C}_{4}, \mathrm{C}_{5}, \mathrm{C}_{11}, \mathrm{C}_{13}$ and $\mathrm{C}_{14}-\mathrm{H}$ ), 3.00-1.20 (m, $8 \mathrm{H}, \mathrm{C}_{7}, \mathrm{C}_{8}, \mathrm{C}_{9}$ and $\left.\mathrm{C}_{10}-\mathrm{CH}_{2}\right) ;{ }^{13} \mathrm{C}$ NMR $\left(\mathrm{CDCl}_{3}\right) \delta$ : $156.1\left(\mathrm{C}_{1 \mathrm{a}}\right), 142.3\left(\mathrm{C}_{2 \mathrm{a}}\right), 137.5\left(\mathrm{C}_{6}\right), 132.2\left(\mathrm{C}_{3}\right), 131.3$ $\left(\mathrm{C}_{15 \mathrm{a}}\right), 130.1\left(\mathrm{C}_{1 \mathrm{~b}}\right), 129.7\left(\mathrm{C}_{7 \mathrm{a}}\right), 129.2\left(\mathrm{C}_{2}\right), 128.3$ $\left(\mathrm{C}_{4}\right), 127.4\left(\mathrm{C}_{11 \mathrm{a}}\right), 126.7\left(\mathrm{C}_{5}\right), 125.6\left(\mathrm{C}_{6 \mathrm{a}}\right), 123.3\left(\mathrm{C}_{12}\right)$, $123.4\left(\mathrm{C}_{11}\right), 123.2\left(\mathrm{C}_{11 \mathrm{~b}}\right), 119.2\left(\mathrm{C}_{13}\right), 112.1\left(\mathrm{C}_{14}\right)$, 
$28.5\left(\mathrm{C}_{7}\right), 27.3\left(\mathrm{C}_{8}\right), 26.9\left(\mathrm{C}_{9}\right), 19.7\left(\mathrm{C}_{10}\right)$; $\mathrm{MS}: \mathrm{m} / \mathrm{z}$ (\%) $366\left(\mathrm{M}^{+} 100 \%\right), 368(\mathrm{M}+2,18 \%)$; Anal. calcd. for $\mathrm{C}_{21} \mathrm{H}_{16} \mathrm{~N}_{2} \mathrm{Cl}_{2}: \mathrm{C}, 68.68 ; \mathrm{H}, 4.39 ; \mathrm{N}, 7.63$. Found: $\mathrm{C}$, 68.70; H, 4.38; N, 7.64\%.

2.2d 6-Chloro-7,8,9,10-tetrahydroquinolino[2', 3': 8,7]cyclooct[b]indole $(\mathbf{8 d})$ : Pale orange solid; yield: $47 \%$; M.p. $190-192^{\circ} \mathrm{C}$ IR $\left(\mathrm{KBr}, \mathrm{cm}^{-1}\right) v_{\max }: 3254$ $(\mathrm{N}-\mathrm{H}), 1588(\mathrm{C}=\mathrm{N}) .{ }^{1} \mathrm{H} \mathrm{NMR}\left(\mathrm{CDCl}_{3}\right) \delta: 9.24$ (b s, $\left.1 \mathrm{H}, \mathrm{N}_{15}-\mathrm{H}\right), 7.78-6.93\left(\mathrm{~m}, 8 \mathrm{H}, \mathrm{C}_{2}, \mathrm{C}_{3}, \mathrm{C}_{4}, \mathrm{C}_{5}, \mathrm{C}_{11}\right.$, $\mathrm{C}_{12}, \mathrm{C}_{13}$ and $\left.\mathrm{C}_{14}-\mathrm{H}\right), 3.31-1.50\left(\mathrm{~m}, 8 \mathrm{H}, \mathrm{C}_{7}, \mathrm{C}_{8}, \mathrm{C}_{9}\right.$ and $\left.\mathrm{C}_{10}-\mathrm{CH}_{2}\right) ;{ }^{13} \mathrm{C}$ NMR $\left(\mathrm{CDCl}_{3}\right) \delta: 156.21\left(\mathrm{C}_{1 \mathrm{a}}\right), 142.26$ $\left(\mathrm{C}_{2 \mathrm{a}}\right), 137.70\left(\mathrm{C}_{6}\right), 132.31\left(\mathrm{C}_{3}\right), 130.94\left(\mathrm{C}_{15 \mathrm{a}}\right), 130.02$ $\left(\mathrm{C}_{1 \mathrm{~b}}\right), 129.59\left(\mathrm{C}_{7 \mathrm{a}}\right), 128.86\left(\mathrm{C}_{2}\right), 128.10\left(\mathrm{C}_{4}\right), 127.35$ $\left(\mathrm{C}_{11 \mathrm{a}}\right), 126.61\left(\mathrm{C}_{5}\right), 125.64\left(\mathrm{C}_{6 \mathrm{a}}\right), 123.54\left(\mathrm{C}_{11 \mathrm{~b}}\right), 122.65$ $\left(\mathrm{C}_{12}\right), 120.02\left(\mathrm{C}_{13}\right), 112.01\left(\mathrm{C}_{14}\right), 115.86\left(\mathrm{C}_{11}\right), 28.34$ $\left(\mathrm{C}_{7}\right), 27.46\left(\mathrm{C}_{8}\right), 26.67\left(\mathrm{C}_{9}\right), 19.09\left(\mathrm{C}_{10}\right)$; $\mathrm{MS}: \mathrm{m} / \mathrm{z}$ (\%) $332\left(\mathrm{M}^{+} 100 \%\right), 334(\mathrm{M}+2,18 \%)$; Anal. calcd. for $\mathrm{C}_{21} \mathrm{H}_{17} \mathrm{~N}_{2} \mathrm{Cl}$ : $\mathrm{C}, 75.78 ; \mathrm{H}, 5.15 ; \mathrm{N}, 8.62$. Found: $\mathrm{C}$, 75.76; H, 5.14; N, 8.63\%.

\subsection{General procedure for the synthesis}

of 7,8,9,10-tetrahydro(pyrazino[2", $\left.3^{\prime \prime}-e^{\prime}\right]$ pyrido)$\left[2^{\prime}, 3^{\prime}: 8,7\right]$ cyclooct $[b]$ indole $(10 a-d)$

A mixture of the appropriate 1-oxo-1,2,3,4,5,6-hexahydrocyclooct $[b]$ indole $(\mathbf{6}, 1 \mathrm{mmol})$, and 3-aminopyrazine-2-carboxylic acid $(1 \mathrm{mmol}) 20 \mathrm{~mL}$ of phosphorous oxychloride was refluxed at $140^{\circ} \mathrm{C}$ for $16 \mathrm{~h}$. The reaction was monitored by using TLC. After the completion of the reaction, it was poured into ice water and then neutralized with sodium bicarbonate solution, extracted with ethyl acetate. The combined organic layers were dried over anhydrous sodium sulphate. It was then purified by column chromatography over silica gel using petroleum ether:ethyl acetate mixture (97:3) and recrystallised from ethanol to yield the respective 6-chloro-7,8,9,10-tetrahydro(pyrazino [2", $3^{\prime \prime}$-']pyrido)$\left[2^{\prime}, 3^{\prime}: 8,7\right]$ cyclooct $[b]$ indole $\mathbf{1 0}$.

2.3a 6-Chloro-12-methyl-7,8,9,10-tetrahydro(pyrazino $\left[2^{\prime \prime}, 3^{\prime \prime}-e^{\prime}\right]$ pyrido)-[2', $\left.3^{\prime}: 8,7\right]$ cyclooct [b]indole (10a): Pale yellow solid; yield: $65 \%$; M.p. $184-186^{\circ} \mathrm{C}$; IR $\left(\mathrm{KBr}, \mathrm{cm}^{-1}\right) v_{\max }: 1613(\mathrm{C}=\mathrm{N}) ;{ }^{1} \mathrm{H} \mathrm{NMR}\left(\mathrm{CDCl}_{3}\right) \delta$ : $9.00\left(\mathrm{~s}, 1 \mathrm{H}, \mathrm{N}_{15}-\mathrm{H}\right), 7.69\left(\mathrm{~d}, 1 \mathrm{H}, \mathrm{C}_{4}-\mathrm{H} J=7.50 \mathrm{~Hz}\right)$, $7.58\left(\mathrm{~d}, 1 \mathrm{H}, \mathrm{C}_{3}-\mathrm{H} J=7.50 \mathrm{~Hz}\right), 7.50-6.95\left(\mathrm{~m}, 3 \mathrm{H}, \mathrm{C}_{11}\right.$, $\mathrm{C}_{13}$ and $\left.\mathrm{C}_{14}-\mathrm{H}\right), 3.25-2.80\left(\mathrm{~m}, 4 \mathrm{H}, \mathrm{C}_{7}, \& \mathrm{C}_{10}-\mathrm{CH}_{2}\right)$, $2.50\left(\mathrm{~s}, 3 \mathrm{H}, \mathrm{C}_{12}-\mathrm{CH}_{3}\right), 2.25-1.50\left(\mathrm{~m}, 4 \mathrm{H}, \mathrm{C}_{8} \& \mathrm{C}_{9}-\right.$ $\left.\mathrm{CH}_{2}\right) ;{ }^{13} \mathrm{C}$ NMR $\left(\mathrm{CDCl}_{3}\right) \delta: 158.1\left(\mathrm{C}_{15 \mathrm{~b}}\right), 148.3\left(\mathrm{C}_{1 \mathrm{a}}\right)$, $146.9\left(\mathrm{C}_{5 \mathrm{a}}\right), 145.2\left(\mathrm{C}_{3}\right), 145.2\left(\mathrm{C}_{4}\right), 143.3\left(\mathrm{C}_{6}\right), 137.7$ $\left(\mathrm{C}_{6 \mathrm{a}}\right), 134.1\left(\mathrm{C}_{14 \mathrm{a}}\right), 132.5\left(\mathrm{C}_{10 \mathrm{~b}}\right), 132.1\left(\mathrm{C}_{12}\right), 123.3$ $\left(\mathrm{C}_{15 \mathrm{a}}\right), 121.5\left(\mathrm{C}_{11}\right), 119.2\left(\mathrm{C}_{13}\right), 113.3\left(\mathrm{C}_{10 \mathrm{a}}\right), 111.4$
$\left(\mathrm{C}_{14}\right), 33.3\left(\mathrm{C}_{9}\right), 31.6\left(\mathrm{C}_{8}\right), 21.3\left(\mathrm{C}_{12}-\mathrm{CH}_{3}\right), 22.9\left(\mathrm{C}_{10}\right)$. $19.6\left(\mathrm{C}_{7}\right)$, MS : m/z (\%) $348\left(\mathrm{M}^{+} 100 \%\right), 350(\mathrm{M}+2$, $18 \%$ ); Anal. Calcd. for $\mathrm{C}_{20} \mathrm{H}_{17} \mathrm{ClN}_{4}$ : C, 68.86; H, 4.91; N, 16.06. Found: C, 68.87; H, 4.93; N, 16.05\%.

2.3b 6-Chloro-14-methyl-7,8,9,10-tetrahydro(pyrazino $\left[2^{\prime \prime}, 3^{\prime \prime}-e^{\prime}\right]$ pyrido)-[2',3':8,7] cyclooct[b]indole (10b): Yellow solid; yield: $65 \%$; M.p. $184-187^{\circ} \mathrm{C}$; IR $(\mathrm{KBr}$, $\left.\mathrm{cm}^{-1}\right) v_{\max }: 1624(\mathrm{C}=\mathrm{N}) ;{ }^{1} \mathrm{H} \mathrm{NMR}\left(\mathrm{CDCl}_{3}\right) \delta: 9.00(\mathrm{~s}$, $\left.1 \mathrm{H}, \mathrm{N}_{15}-\mathrm{H}\right), 7.45\left(\mathrm{~d}, 1 \mathrm{H}, \mathrm{C}_{4}-\mathrm{H} J=7.50 \mathrm{~Hz}\right), 7.38(\mathrm{~d}$, $\left.1 \mathrm{H}, \mathrm{C}_{3}-\mathrm{HJ}=7.50 \mathrm{~Hz}\right), 7.35-6.98\left(\mathrm{~m}, 3 \mathrm{H}, \mathrm{C}_{11}, \mathrm{C}_{12}\right.$ and $\left.\mathrm{C}_{13}-\mathrm{H}\right), 3.25-1.50\left(\mathrm{~m}, 8 \mathrm{H}, \mathrm{C}_{7}, \mathrm{C}_{8}, \mathrm{C}_{9}\right.$ and $\left.\mathrm{C}_{10^{-}}-\mathrm{CH}_{2}\right)$, $2.50\left(\mathrm{~s}, 3 \mathrm{H}, \mathrm{C}_{14}-\mathrm{CH}_{3}\right) ;{ }^{13} \mathrm{C} \mathrm{NMR}\left(\mathrm{CDCl}_{3}\right) \delta: 158.34$ $\left(\mathrm{C}_{15 \mathrm{~b}}\right), 148.30\left(\mathrm{C}_{1 \mathrm{a}}\right), 146.79\left(\mathrm{C}_{5 \mathrm{a}}\right), 145.32\left(\mathrm{C}_{3}\right), 145.25$ $\left(\mathrm{C}_{4}\right), 143.32\left(\mathrm{C}_{6}\right), 137.63\left(\mathrm{C}_{6 \mathrm{a}}\right), 134.12\left(\mathrm{C}_{14 \mathrm{a}}\right), 132.25$ $\left(\mathrm{C}_{10 \mathrm{~b}}\right), 132.14\left(\mathrm{C}_{12}\right), 123.40\left(\mathrm{C}_{15 \mathrm{a}}\right), 121.64\left(\mathrm{C}_{11}\right)$, $119.43\left(\mathrm{C}_{13}\right), 113.12\left(\mathrm{C}_{10 \mathrm{a}}\right), 111.49\left(\mathrm{C}_{14}\right), 33.39\left(\mathrm{C}_{9}\right)$, $31.50\left(\mathrm{C}_{8}\right), 22.30\left(\mathrm{C}_{14}-\mathrm{CH}_{3}\right), 22.29\left(\mathrm{C}_{10}\right)$; $\mathrm{MS}: \mathrm{m} / \mathrm{z}$ (\%) $348\left(\mathrm{M}^{+} 100 \%\right), 350(\mathrm{M}+2,18 \%)$; Anal. Calcd. for $\mathrm{C}_{20} \mathrm{H}_{17} \mathrm{ClN}_{4}$ : C, 68.86; H, 4.91; N, 16.06. Found: C, $68.87 ; \mathrm{H}, 4.93 ; \mathrm{N}, 16.05 \%$.

2.3c 6,12-Dichloro-7, 8,9,10-tetrahydro(pyrazino $\left[2^{\prime \prime}, 3^{\prime \prime}-e^{\prime}\right]$ pyrido $)-\left[2^{\prime}, 3^{\prime}: 8,7\right]$ cyclooct [b]indole (10c): Brown yellow solid; yield: $65 \%$; M.p. $189-191^{\circ} \mathrm{C}$; IR $\left(\mathrm{KBr}, \mathrm{cm}^{-1}\right) v_{\max }: 1635(\mathrm{C}=\mathrm{N}) ;{ }^{1} \mathrm{H}$ NMR $\left(\mathrm{CDCl}_{3}\right) \delta$ : $9.00\left(\mathrm{~s}, 1 \mathrm{H}, \mathrm{N}_{15}-\mathrm{H}\right), 7.60\left(\mathrm{~d}, 1 \mathrm{H}, \mathrm{C}_{4}-\mathrm{H} J=7.50 \mathrm{~Hz}\right)$, $7.70\left(\mathrm{~d}, 1 \mathrm{H}, \mathrm{C}_{3}-\mathrm{H}, J=7.50 \mathrm{~Hz}\right), 6.95-7.45(\mathrm{~m}, 3 \mathrm{H}$, $\mathrm{C}_{11}, \mathrm{C}_{13}$ and $\left.\mathrm{C}_{14}-\mathrm{H}\right), 3.35-1.50\left(\mathrm{~m}, 8 \mathrm{H}, \mathrm{C}_{7}, \mathrm{C}_{8}, \mathrm{C}_{9}\right.$ and $\left.\mathrm{C}_{10}-\mathrm{CH}_{2}\right) ;{ }^{13} \mathrm{C}$ NMR $\left(\mathrm{CDCl}_{3}\right) \quad \delta: 157.2\left(\mathrm{C}_{15 \mathrm{~b}}\right), 147.7$ $\left(\mathrm{C}_{1 \mathrm{a}}\right), 146.4\left(\mathrm{C}_{5 \mathrm{a}}\right), 145.8\left(\mathrm{C}_{3}\right), 145.2\left(\mathrm{C}_{4}\right), 143.9\left(\mathrm{C}_{6}\right)$, $137.6\left(\mathrm{C}_{6 \mathrm{a}}\right), 134.2\left(\mathrm{C}_{14 \mathrm{a}}\right), 132.5\left(\mathrm{C}_{10 \mathrm{~b}}\right), 132.4\left(\mathrm{C}_{12}\right)$, $123.4\left(\mathrm{C}_{15 \mathrm{a}}\right), 121.6\left(\mathrm{C}_{11}\right), 119.4\left(\mathrm{C}_{13}\right), 113.2\left(\mathrm{C}_{10 \mathrm{a}}\right)$, $111.9\left(\mathrm{C}_{14}\right), 33.9\left(\mathrm{C}_{9}\right), 31.5\left(\mathrm{C}_{8}\right), 22.2\left(\mathrm{C}_{10}\right)$; $\mathrm{MS}: \mathrm{m} / \mathrm{z}$ (\%) $369\left(\mathrm{M}^{+} 100 \%\right), 371(\mathrm{M}+2,18 \%)$; Anal. Calcd. for $\mathrm{C}_{19} \mathrm{H}_{14} \mathrm{Cl}_{2} \mathrm{~N}_{4}$ : C, 61.80; H, 3.82; N, 15.17. Found: C, $61.82 ; \mathrm{H}, 3.79 ; \mathrm{N}, 15.15 \%$.

2.3d 6-Chloro-7,8,9,10-tetrahydro(pyrazino [2",3"-e'] pyrido)-[2', $\left.3^{\prime}: 8,7\right]$ cyclooct[b]indole $(\mathbf{1 0 d})$ : Orange yellow solid; yield: $65 \%$; M.p. $186-188^{\circ} \mathrm{C}$; IR $(\mathrm{KBr}$, $\left.\mathrm{cm}^{-1}\right) v_{\max }: 1605(\mathrm{C}=\mathrm{N}) ;{ }^{1} \mathrm{H}$ NMR $\left(\mathrm{CDCl}_{3}\right) \delta: 9.10$ (s, $\left.1 \mathrm{H}, \mathrm{N}_{15}-\mathrm{H}\right), 7.45\left(\mathrm{~d}, 1 \mathrm{H}, \mathrm{C}_{4}-\mathrm{H} J=7.00 \mathrm{~Hz}\right), 7.35$ $\left(\mathrm{d}, 1 \mathrm{H}, \mathrm{C}_{3}, J=7.00 \mathrm{~Hz}\right), 6.90-7.35\left(\mathrm{~m}, 4 \mathrm{H}, \mathrm{C}_{11}\right.$, $\mathrm{C}_{12}, \mathrm{C}_{13}$ and $\left.\mathrm{C}_{14}-\mathrm{H}\right), 3.40-2.98\left(\mathrm{~m}, 4 \mathrm{H}, \mathrm{C}_{7} \& \mathrm{C}_{10^{-}}\right.$ $\left.\mathrm{CH}_{2}\right), 2.25-1.45\left(\mathrm{~m}, 4 \mathrm{H}, \mathrm{C}_{8}, \& \mathrm{C}_{9}-\mathrm{CH}_{2}\right) ;{ }^{13} \mathrm{C} \mathrm{NMR}$ $\left(\mathrm{CDCl}_{3}\right) \delta: 157.4\left(\mathrm{C}_{15 \mathrm{~b}}\right), 147.5\left(\mathrm{C}_{1 \mathrm{a}}\right), 146.7\left(\mathrm{C}_{5 \mathrm{a}}\right), 145.2$ $\left(\mathrm{C}_{3}\right), 145.1\left(\mathrm{C}_{4}\right), 143.5\left(\mathrm{C}_{6}\right), 137.7\left(\mathrm{C}_{6 \mathrm{a}}\right), 134.5\left(\mathrm{C}_{14 \mathrm{a}}\right)$, $132.6\left(\mathrm{C}_{10 \mathrm{~b}}\right), 132.7\left(\mathrm{C}_{12}\right), 123.6\left(\mathrm{C}_{15 \mathrm{a}}\right), 121.2\left(\mathrm{C}_{11}\right)$, $119.1\left(\mathrm{C}_{13}\right), 113.6\left(\mathrm{C}_{10 \mathrm{a}}\right), 111.7\left(\mathrm{C}_{14}\right), 33.4\left(\mathrm{C}_{9}\right), 31.2$ $\left(\mathrm{C}_{8}\right), 22.4\left(\mathrm{C}_{10}\right)$; $\mathrm{MS}: \mathrm{m} / \mathrm{z}(\%) 334\left(\mathrm{M}^{+} 100 \%\right), 336$ 
$(\mathrm{M}+2,18 \%)$; Anal. Calcd. for $\mathrm{C}_{19} \mathrm{H}_{15} \mathrm{ClN}_{4}$ : C, 68.16; H, 4.52; N, 16.73. Found: C, 68.17; H, 4.50; N, $16.74 \%$.

2.4 General procedure for the synthesis of 7,8,9,10tetrahydroquinolino $\left[2^{\prime}, 3^{\prime}: 8,7\right]$ cyclooct [b]indole- 6 carboxylic acid $(11 a-d)$

A mixture of the respective 1-oxo-1,2,3,4,5,6-hexahydrocyclooct $[b]$ indole $(\mathbf{6}, 1 \mathrm{mmol})$, isatin $(1 \mathrm{mmol})$ and $\mathrm{NaOH}(0.400 \mathrm{~g})$ in ethanol $(15 \mathrm{~mL})$ was refluxed in a steam bath for $24 \mathrm{~h}$. The reaction was monitored by TLC. After completion of the reaction, the excess solvent was removed and poured into ice and neutralized with dilute $\mathrm{HCl}$. The crude solid obtained was filtered and purified with sodium bicarbonate treatment and neutralized with dilute $\mathrm{HCl}$. The solid product was recrystalized from ethanol to yield the respective 7,8,9,10-tetrahydroquinolino[ $\left[2^{\prime}, 3^{\prime}\right.$ : 8,7]cyclooct[b]indole-6-carboxylic acid $\mathbf{1 1}$.

2.4a 12-Methyl-7,8,9,10-tetrahydroquinolino[2', 3': 8,7]cyclooct[b]indole-6-carboxylic acid (11a): Orange solid; yield: $85 \%$ M.p. $>300^{\circ} \mathrm{C}$ IR $\left(\mathrm{KBr}, \mathrm{cm}^{-1}\right) v_{\max }$ :, $3400(\mathrm{O}-\mathrm{H}), 1712(\mathrm{C}=\mathrm{O}), 1605(\mathrm{C}=\mathrm{N}) ;{ }^{1} \mathrm{H}$ NMR (DMSO) $\delta: 11.40(\mathrm{~b} \mathrm{~s}, 1 \mathrm{H}$, acid $\mathrm{OH}), 9.20(\mathrm{~b} \mathrm{~s}, 1 \mathrm{H}$, $\left.\mathrm{N}_{15}-\mathrm{H}\right)$. 8.00-7.70 (m, 2H, $\mathrm{C}_{3}$ and $\left.\mathrm{C}_{4}-\mathrm{H}\right), 7.39-7.29$ $\left(\mathrm{m}, 3 \mathrm{H}, \mathrm{C}_{2}, \mathrm{C}_{5}\right.$ and $\left.\mathrm{C}_{11}-\mathrm{H}\right), 7.20-6.95\left(\mathrm{~m}, 2 \mathrm{H}, \mathrm{C}_{13}\right.$ and $\left.\mathrm{C}_{14}-\mathrm{H}\right)$ 3.30-3.10 (m, 4H, $\left.\mathrm{C}_{7} \& \mathrm{C}_{10}-\mathrm{CH}_{2}\right), 2.50(\mathrm{~s}$, $\left.3 \mathrm{H}, \mathrm{C}_{14}-\mathrm{CH}_{3}\right) ; 2.85-1.80\left(\mathrm{~m}, 4 \mathrm{H}, \mathrm{C}_{8} \& \mathrm{C}_{9}-\mathrm{CH}_{2}\right) ;{ }^{13} \mathrm{C}$ NMR (DMSO) $\delta: 172.00\left(\mathrm{C}_{6}-\mathrm{COOH}\right), 157.54\left(\mathrm{C}_{1 \mathrm{a}}\right)$, $152.67\left(\mathrm{C}_{6}\right), 146.76\left(\mathrm{C}_{2 \mathrm{a}}\right), 132.90\left(\mathrm{C}_{7 \mathrm{a}}\right), 130.21\left(\mathrm{C}_{1 \mathrm{~b}}\right)$, $130.04\left(\mathrm{C}_{15 \mathrm{a}}\right), 128.78\left(\mathrm{C}_{2}\right), 127.47\left(\mathrm{C}_{3}\right), 126.95\left(\mathrm{C}_{11 \mathrm{a}}\right)$, $126.78\left(\mathrm{C}_{4}\right), 125.12\left(\mathrm{C}_{12}\right), 124.38\left(\mathrm{C}_{5}\right), 123.15\left(\mathrm{C}_{11}\right)$, $122.26\left(\mathrm{C}_{6 \mathrm{a}}\right), 120.05\left(\mathrm{C}_{13}\right), 114.87\left(\mathrm{C}_{11 \mathrm{~b}}\right), 111.54\left(\mathrm{C}_{14}\right)$, $32.32\left(\mathrm{C}_{9}\right), 31.54\left(\mathrm{C}_{8}\right), 22.75\left(\mathrm{C}_{10}\right), 22.43\left(\mathrm{C}_{7}\right), 21.74$ $\left(\mathrm{C}_{12}-\mathrm{CH}_{3}\right) . \mathrm{MS}: \mathrm{m} / \mathrm{z}(\%) 356\left(\mathrm{M}^{+} 100 \%\right), 358(\mathrm{M}+2$, $18 \%$ ); Anal. calcd. for $\mathrm{C}_{23} \mathrm{H}_{20} \mathrm{~N}_{2} \mathrm{O}_{2}: \mathrm{C}, 77.51 ; \mathrm{H}, 5.66$; N, 7.86; Found: C, 77.49; H, 5.67; N, 7.88\%.

2.4b 14-Methyl-7,8,9,10-tetrahydroquinolino[2', 3': 8,7]cyclooct[b]indole-6-carboxylic acid (11b): Yellow solid; yield: $80 \%$; M.p. $>300^{\circ} \mathrm{C}$ IR $(\mathrm{KBr}$, $\left.\mathrm{cm}^{-1}\right) v_{\max }: 3449(\mathrm{O}-\mathrm{H}), 1705(\mathrm{C}=\mathrm{O}), 1614(\mathrm{C}=\mathrm{N})$; ${ }^{1} \mathrm{H}$ NMR (DMSO) $\delta: 11.23(\mathrm{~s}, 1 \mathrm{H}$, acid $\mathrm{OH}), 9.18(\mathrm{~b}$ s, $\left.\mathrm{N}_{15}-\mathrm{H}\right)$. 7.85-7.78 (m, 2H, $\mathrm{C}_{3}$ and $\left.\mathrm{C}_{4}-\mathrm{H}\right), 7.47-7.30$ $\left(\mathrm{m}, 3 \mathrm{H}, \mathrm{C}_{2}, \mathrm{C}_{5}\right.$ and $\left.\mathrm{C}_{11}-\mathrm{H}\right), 7.10-7.05\left(\mathrm{~m}, 2 \mathrm{H}, \mathrm{C}_{12}\right.$ \& $\left.\mathrm{C}_{13}-\mathrm{H}\right), 3.30-3.05\left(\mathrm{~m}, 4 \mathrm{H}, \mathrm{C}_{7} \& \mathrm{C}_{10}-\mathrm{CH} 2\right), 2.50(\mathrm{~s}$, $\left.3 \mathrm{H}, \mathrm{C}_{14}-\mathrm{CH}_{3}\right), 2.15-1.80\left(\mathrm{~m}, 4 \mathrm{H}, \mathrm{C}_{8} \& \mathrm{C}_{9}-\mathrm{CH}_{2}\right) ;{ }^{13} \mathrm{C}$ NMR (DMSO) $\delta: 173.12\left(\mathrm{C}_{6}-\mathrm{COOH}\right), 157.56\left(\mathrm{C}_{1 \mathrm{a}}\right)$, $152.76\left(\mathrm{C}_{6}\right), 147.10\left(\mathrm{C}_{2 \mathrm{a}}\right), 132.34\left(\mathrm{C}_{7 \mathrm{a}}\right), 131.06\left(\mathrm{C}_{1 \mathrm{~b}}\right)$, $130.86\left(\mathrm{C}_{15 \mathrm{a}}\right), 128.57\left(\mathrm{C}_{2}\right), 127.68\left(\mathrm{C}_{3}\right), 126.86\left(\mathrm{C}_{4 \mathrm{a}}\right)$, $126.10\left(\mathrm{C}_{4}\right), 124.54\left(\mathrm{C}_{5}\right), 122.44\left(\mathrm{C}_{6 \mathrm{a}}\right), 122.18\left(\mathrm{C}_{11}\right)$,
$121.11\left(\mathrm{C}_{12}\right), 120.89\left(\mathrm{C}_{13}\right), 115.05\left(\mathrm{C}_{11 \mathrm{~b}}\right), 113.31\left(\mathrm{C}_{14}\right)$, $32.65\left(\mathrm{C}_{9}\right), 31.23\left(\mathrm{C}_{8}\right), 22.34\left(\mathrm{C}_{10}\right), 22.15\left(\mathrm{C}_{7}\right) 16.50$ $\left(\mathrm{C}_{14}-\mathrm{CH}_{3}\right)$. MS : m/z (\%) $356\left(\mathrm{M}^{+} 100 \%\right), 358(\mathrm{M}+2$, $18 \%$ ); Anal. calcd. for $\mathrm{C}_{23} \mathrm{H}_{20} \mathrm{~N}_{2} \mathrm{O}_{2}: \mathrm{C}, 77.51 ; \mathrm{H}, 5.66$; N, 7.86; Found: C, 77.49; H, 5.67; N, 7.88\%.

$2.4 \mathrm{c}$ 12-Chloro-7,8,9,10-tetrahydroquinolino [2', $3^{\prime}$ : 8,7]cyclooct[b]indole-6-carboxylic acid (11c): Brown solid; yield: $82 \%$; M.p. $>300^{\circ} \mathrm{C}$ IR $(\mathrm{KBr}$, $\left.\mathrm{cm}^{-1}\right) v_{\max }$ : $3434(\mathrm{O}-\mathrm{H}), 1720(\mathrm{C}=\mathrm{O}), 1602(\mathrm{C}=\mathrm{N})$;. ${ }^{1} \mathrm{H}$ NMR (DMSO) $\delta: 11.15$ (b s, $1 \mathrm{H}$, acid $\mathrm{OH}$ ), 9.20 (b s, $\left.1 \mathrm{H}, \mathrm{N}_{15}-\mathrm{H}\right)$ 7.98-7.80 (m, 2H, $\mathrm{C}_{3}$ and $\mathrm{C}_{4}-\mathrm{H}$ ), $7.52-7.29\left(\mathrm{~m}, 3 \mathrm{H}, \mathrm{C}_{2}, \mathrm{C}_{5}\right.$ and $\left.\mathrm{C}_{11}-\mathrm{H}\right), 7.20-7.10(\mathrm{~m}$, $2 \mathrm{H}, \mathrm{C}_{13}$ and $\left.\mathrm{C}_{14}-\mathrm{H}\right), 3.20-1.78\left(\mathrm{~m}, 8 \mathrm{H}, \mathrm{C}_{7}, \mathrm{C}_{8}, \mathrm{C}_{9}\right.$ and $\left.\mathrm{C}_{10}-\mathrm{CH}_{2}\right) .{ }^{13} \mathrm{C}$ NMR (DMSO) $\delta: 174.10\left(\mathrm{C}_{6}-\mathrm{COOH}\right)$, $156.89\left(\mathrm{C}_{1 \mathrm{a}}\right), 152.14\left(\mathrm{C}_{6}\right), 147.64\left(\mathrm{C}_{2 \mathrm{a}}\right), 132.27\left(\mathrm{C}_{7 \mathrm{a}}\right)$, $132.14\left(\mathrm{C}_{1 \mathrm{~b}}\right), 131.02\left(\mathrm{C}_{15 \mathrm{a}}\right), 128.89\left(\mathrm{C}_{2}\right), 127.98\left(\mathrm{C}_{3}\right)$, $127.12\left(\mathrm{C}_{11 \mathrm{a}}\right), 126.24\left(\mathrm{C}_{4}\right), 125.03\left(\mathrm{C}_{5}\right), 124.13\left(\mathrm{C}_{12}\right)$, $122.53\left(\mathrm{C}_{6 \mathrm{a}}\right), 122.54\left(\mathrm{C}_{11}\right), 120.11\left(\mathrm{C}_{13}\right), 115.50\left(\mathrm{C}_{11 \mathrm{~b}}\right)$, $113.89\left(\mathrm{C}_{14}\right), 32.34\left(\mathrm{C}_{9}\right), 31.76\left(\mathrm{C}_{8}\right), 22.65\left(\mathrm{C}_{10}\right), 22.54$ $\left(\mathrm{C}_{7}\right) . \mathrm{MS}: \mathrm{m} / \mathrm{z}(\%) 375\left(\mathrm{M}^{+} 100 \%\right), 377(\mathrm{M}+2,18 \%)$; Anal. calcd. for $\mathrm{C}_{22} \mathrm{H}_{17} \mathrm{~N}_{2} \mathrm{O}_{2} \mathrm{Cl}: \mathrm{C}, 70.12 ; \mathrm{H}, 4.55 ; \mathrm{N}$, 7.43; Found: C, 70.14; H, 4.53 N 7.45\%.

$2.4 \mathrm{~d} 7,8,9,10$-Tetrahydroquinolino $\left[2^{\prime}, 3^{\prime}: 8,7\right]$ cyclooct [b]indole-6-carboxylic acid (11d): Pale yellow solid; yield: $78 \%$; M.p. $>300^{\circ} \mathrm{C}$ IR $\left(\mathrm{KBr}, \mathrm{cm}^{-1}\right) v_{\max }: 3272$ $(\mathrm{O}-\mathrm{H}), 1727(\mathrm{C}=\mathrm{O}), 1632(\mathrm{C}=\mathrm{N}) ; .{ }^{1} \mathrm{H}$ NMR $(\mathrm{DMSO})$ $\delta: 11.10$ (s, $1 \mathrm{H}$, acid-OH). 8.99 (b s, $\left.1 \mathrm{H}, \mathrm{N}_{15}-\mathrm{H}\right), 7.80$ $7.69\left(\mathrm{~m}, 2 \mathrm{H}, \mathrm{C}_{3}\right.$ and $\left.\mathrm{C}_{4}-\mathrm{H}\right), 7.60-7.32\left(\mathrm{~m}, 3 \mathrm{H}, \mathrm{C}_{2}, \mathrm{C}_{5}\right.$ and $\left.\mathrm{C}_{11}-\mathrm{H}\right), 7.20-7.00\left(\mathrm{~m}, 3 \mathrm{H}, \mathrm{C}_{12}, \mathrm{C}_{13}\right.$ and $\left.\mathrm{C}_{14}-\mathrm{H}\right)$, 3.35-1.80 (m, 8H, $\mathrm{C}_{7}, \mathrm{C}_{8}, \mathrm{C}_{9}$ and $\left.\mathrm{C}_{10}-\mathrm{CH}_{2}\right) ;{ }^{13} \mathrm{C} \mathrm{NMR}$ (DMSO) $\delta: 173.79\left(\mathrm{C}_{6}-\mathrm{COOH}\right), 156.75\left(\mathrm{C}_{1 \mathrm{a}}\right), 152.27$ $\left(\mathrm{C}_{6}\right), 147.29\left(\mathrm{C}_{2 \mathrm{a}}\right), 136.89\left(\mathrm{C}_{7 \mathrm{a}}\right), 132.89\left(\mathrm{C}_{1 \mathrm{~b}}\right), 131.19$ $\left(\mathrm{C}_{15 \mathrm{a}}\right), 128.90\left(\mathrm{C}_{2}\right), 127.65\left(\mathrm{C}_{3}\right), 127.18\left(\mathrm{C}_{11 \mathrm{a}}\right), 126.83$ $\left(\mathrm{C}_{4}\right), 125.42\left(\mathrm{C}_{5}\right), 122.32\left(\mathrm{C}_{6 \mathrm{a}}\right), 121.72\left(\mathrm{C}_{11}\right), 119.67$ $\left(\mathrm{C}_{12}\right), 118.68\left(\mathrm{C}_{13}\right), 115.64\left(\mathrm{C}_{11 \mathrm{~b}}\right), 113.72\left(\mathrm{C}_{14}\right), 32.14$ $\left(\mathrm{C}_{9}\right), 31.54\left(\mathrm{C}_{7}\right), 22.89\left(\mathrm{C}_{10}\right) .22 .32\left(\mathrm{C}_{7}\right) . \mathrm{MS}: \mathrm{m} / \mathrm{z}$ (\%) $342\left(\mathrm{M}^{+} 100 \%\right), 344(\mathrm{M}+2,18 \%)$; Anal. calcd. for $\mathrm{C}_{22} \mathrm{H}_{18} \mathrm{~N}_{2} \mathrm{O}_{2}$ : C, 77.17; H, 5.30; N, 8.18; Found: C, 77.16; H, 5.31, N 8.21\%.

\section{Results and Discussion}

In this paper we have have developed a facile process for a highly efficient assembly of cyclooct $[b]$ indole with quinoline and pyrazino pyrido moiety under $\mathrm{POCl}_{3}$ condition and via Pfitzinger reaction in which 1-oxo1,2,3,4,5,6-hexahydrocyclooct $[b]$ indoles $\mathbf{6}$ were used as a potential precursors.

In order to obtain 6-chloro-7,8,9,10-tetrahydroquinolino $\left[2^{\prime}, 3^{\prime}: 8,7\right]$ cyclooct $[b]$ indole 8,1 -oxo-1,2,3,4, 
5,6-hexahydrocyclooct $[b]$ indole 6 was refluxed with anthranilic acid under toluene and $p$-TsOH/ $\mathrm{POCl}_{3}$ condition. The direct condensation of 1-oxo-1,2,3, 4,5,6-hexahydrocyclooct $[b]$ indole $(\mathbf{6}, 0.001 \mathrm{~mol})$ with anthranilic acid $(0.001 \mathrm{~mol})$ under toluene and $p$ $\mathrm{TsOH} / \mathrm{POCl}_{3}$ did not lead to the expected quinolino[ $2^{\prime}$, $\left.3^{\prime}: 8,7\right]$ cyclooct $[b]$ indole $\mathbf{8}$. When the same reaction was carried under phosphorous oxychloride condition at $140^{\circ} \mathrm{C}$ for $16 \mathrm{~h}$ the targeted product 6-chloro-7,8,9,10tetrahydroquinolino $\left[2^{\prime}, 3^{\prime}: 8,7\right]$ cyclooct $[b]$ indole 8 was obtained (scheme 1).

The disappearance of carbonyl stretching frequency and the presence of $-\mathrm{C}=\mathrm{N}$ at $1572 \mathrm{~cm}^{-1}$ in its IR spectrum revealed the formation of 8 . In its ${ }^{1} \mathrm{H}-\mathrm{NMR}$ spectrum the appearance of $\mathrm{N}_{15}-\mathrm{H}$ signal at $\delta 9.00$, seven protons in the aromatic region at $\delta 8.30-7.15$, eight protons of four methylene group $\left(\mathrm{C}_{7}, \mathrm{C}_{8}, \mathrm{C}_{9}, \mathrm{C}_{10}\right)$ at $\delta 3.50-1.00$ and a singlet for $\mathrm{C}_{12}$ methyl proton at $\delta 2.50$, respectively. Its ${ }^{13} \mathrm{C}$ spectrum revealed the presence of 22 carbon atoms. Spectral and analytical data of 8a reported in experimental section of this paper fully support the structure assigned to it. From the spectral and analytical data, the obtained compounds was confirmed as 6-chloro-12-methyl-7,8,9,10tetrahydroquinolino $\left[2^{\prime}, 3^{\prime}: 8,7\right]$ cyclooct $[b]$ indole $(\mathbf{8 a})$. A similar series of compounds were derived from $\mathbf{6 b}-\mathbf{d}$ to yield $\mathbf{8 b}-\mathbf{d}$. Similarly, the yield, mp, spectral data

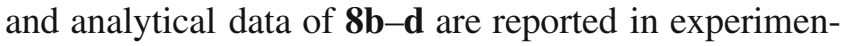
tal section of this paper, fully support the structures assigned to them.

\subsection{Mechanism for the formation of product 8}

A plausible mechanism for the formation of product $\mathbf{8}$ is depicted in (scheme 4). ${ }^{20}$ The compound 1-oxo-1,2,3,4,5,6-hexahydrocyclooct[ $b]$ indole $\mathbf{6}$ underwent phosphorous oxychloride acid catalysed condensation with anthranilic acid to give an intermediate (I) which was in equilibrium with the intermediate $\mathrm{N}$-aryl enamine form (II). The reaction of excess phosphorous oxychloride with the intermediate I gave the mixed anhydride intermediate (III). The intramolecular electrophilic substitution reaction at the second position of the cyclooct[b]indole intermediate facilitated by the lone pair electron on nitrogen of the $\mathrm{N}$-aryl moiety, with the aryl mixed anhydride carbonyl group afforded the intermediate (IV). On subsequent prototropic shift, dehydrogenation and chlorination followed by $\mathrm{PO}_{2} \mathrm{Cl}$ elimination yielded the final product 6-chloro-7,8, 9,10-tetrahydroquinolino[ $\left[2^{\prime}, 3^{\prime}: 8,7\right]$ cyclooct $[b]$ indole $\mathbf{8}$ (scheme 4 ).

In an attempt to extend this synthesis, 1-oxo-1,2, 3,4,5,6-hexahydrocyclooct $[b]$ indole 6 was reacted with 3-aminopyrazine-2-carboxylic acid under $\mathrm{POCl}_{3}$ condition at $140^{\circ} \mathrm{C}$ for $16 \mathrm{~h}$ to afford 6-chloro-7,8,9,10tetrahydro(pyrazino $\left[2^{\prime \prime}, 3^{\prime \prime}\right.$-e']pyrido)-[2', $\left.3^{\prime}: 8,7\right]$ cyclooct $[b]$ indole 10. A mixture of the appropriate 1-oxo1,2,3,4,5,6-hexahydrocyclooct[ $b]$ indole $(\mathbf{6}, 0.001 \mathrm{~mol})$ and 3-aminopyrazine-2-carboxylic acid $(0.001 \mathrm{~mol})$ in $20 \mathrm{~mL}$ of phosphorous oxychloride was refluxed at $140^{\circ} \mathrm{C}$ for $16 \mathrm{~h}$.

In its IR spectrum $\mathrm{C}=\mathrm{N}$ stretching was observed at $1613 \mathrm{~cm}^{-1}$. The ${ }^{1} \mathrm{H}$ NMR spectrum exhibited a broad singlet at $\delta 9.00$ corroborating the presence an indole $\mathrm{NH}$ moiety. The presence of $\mathrm{C}_{3}$ and $\mathrm{C}_{4}$ protons are indicated by two doublets in the region $\delta 7.69$ and 7.58 with $J=8.00 \mathrm{~Hz}$. A cluster of multiplets between $\delta$ 7.50-6.95 was due to $\mathrm{C}_{11}, \mathrm{C}_{13}$ and $\mathrm{C}_{14}-\mathrm{H}$. A cluster of multiplets between $\delta 3.25-1.50$ was due to $\mathrm{C}_{7}, \mathrm{C}_{8}, \mathrm{C}_{9}$ and $\mathrm{C}_{10}-\mathrm{H}$. A singlet at $\delta 2.50$ indicates the presence of $\mathrm{C}_{12}-\mathrm{CH}_{3}$. Its ${ }^{13} \mathrm{C}$ NMR spectrum confirmed the presence of 17 carbons. From the spectral and analytical data, the obtained compounds were confirmed as 6chloro-12-methyl-7,8,9,10-tetrahydro(pyrazino [2" $2^{\prime \prime}$ $\left.\mathrm{e}^{\prime}\right]$ pyrido)- $\left[2^{\prime}, 3^{\prime}: 8,7\right]$ cyclooct $[b]$ indole (10a). A similar series of compounds were derived from $\mathbf{6 b}-\mathbf{d}$ to yield 10b-d.

The synthesis of the targeted tetrahydroquinolino $\left[2^{\prime}, 3^{\prime}: 8,7\right]$ cyclooct $[b]$ indole-6-carboxylic acid $\mathbf{1 1}$ was carried out as outlined in scheme $5 .{ }^{21}$ The versatile Pfitzinger reaction was utilized to synthesize the tetrahydroquinolino $\left[2^{\prime}, 3^{\prime}: 8,7\right]$ cyclooct $[b]$ indole-6-carboxylic acid $\mathbf{1 1}$ in satisfactory yields by reacting isatin with 1-oxo-1,2,3,4,5,6-hexahydrocyclooct $[b]$ indole $\mathbf{6}$. The Pfitzinger reaction (also known as the PfitzingerBorsche reaction) is the chemical reaction of isatin with base and a carbonyl compound to yield substituted carboxylic acids. The reaction proceeded in ethanol in the presence of $\mathrm{KOH}$. The product was obtained only in moderate yield. The best result was obtained when the reaction was performed in the presence of $\mathrm{NaOH}$ instead of $\mathrm{KOH}$. The yield of the products $\mathbf{1 1}$ thus obtained was compared in table 1. Inorder to get tetrahydroquinolino $\left[2^{\prime}, 3^{\prime}: 8,7\right]$ cyclooct $[b]$ indole-6carboxylic acid 11, a mixture of the respective 1-oxo1,2,3,4,5,6-hexahydrocyclooct $[b]$ indole $(\mathbf{6}, 1 \mathrm{mmol})$ was reacted with isatin $(1 \mathrm{mmol})$ and $\mathrm{NaOH}(0.400 \mathrm{~g})$ in ethanol $(20 \mathrm{~mL})$ was refluxed in a steam bath for $24 \mathrm{~h}$.

Its IR spectrum shows absorptions at 3400, 1712 and $1605 \mathrm{~cm}^{-1}$ due to the presence of carboxylic acid $\mathrm{OH}$, $-\mathrm{C}=\mathrm{O}$ and $-\mathrm{C}=\mathrm{N}$, respectively. Its ${ }^{1} \mathrm{H}$ NMR spectrum showed the carboxylic acid $\mathrm{OH}$ at $\delta 11.40$. A broad singlet at $\delta 9.20$ was due to the presence of indole $\mathrm{NH}$. A cluster of multiplet between $\delta 8.00-7.70$ was due to the presence of $\mathrm{C}_{3}$ and $\mathrm{C}_{4}-\mathrm{H}$. A multiplet between $\delta 7.39$ and 7.29 was due to the presence of $\mathrm{C}_{4}, \mathrm{C}_{5}$ and $\mathrm{C}_{11}-\mathrm{H}$. 


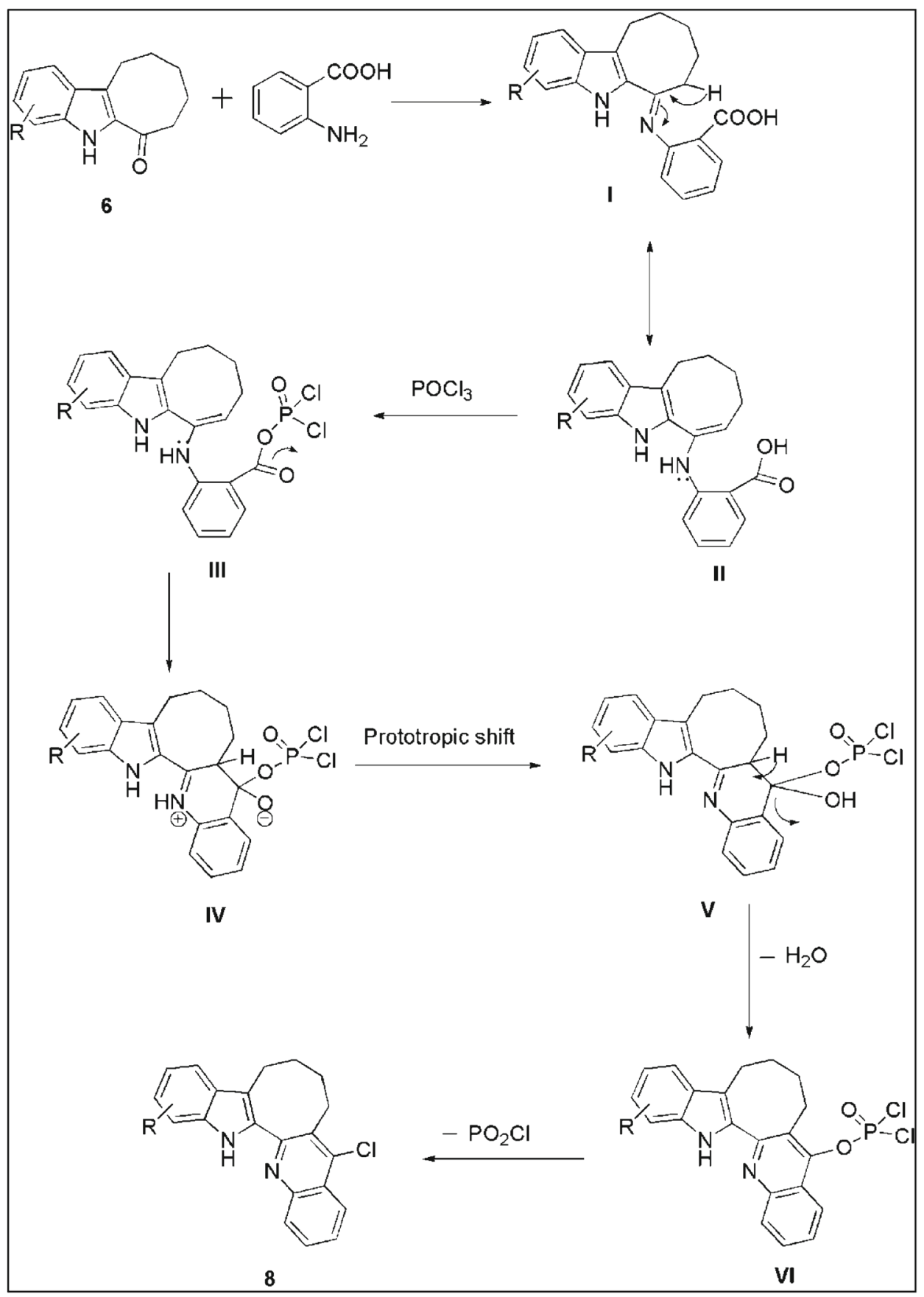

Scheme 4. Mechanism for the formation of product 8 .

A multiplet between $\delta 7.20$ and 6.95 was due to the presence of $\mathrm{C}_{13}$ and $\mathrm{C}_{14}-\mathrm{H}$. A multiplet between $\delta 3.30$ and 1.80 was due to the presence of $\mathrm{C}_{7}, \mathrm{C}_{8}, \mathrm{C}_{9}$ and $\mathrm{C}_{10^{-}}$ $\mathrm{H}$. A singlet at $\delta 2.56$ was due to the presence of $\mathrm{C}_{12^{-}}$ $\mathrm{CH}_{3}$, respectively. Its ${ }^{13} \mathrm{C}$ NMR spectrum confirmed the presence of 23 carbons.

From the spectral and analytical data, the obtained compounds were confirmed as 12-methyl-7,8,9,10tetrahydroquinolino $\left[2^{\prime}, 3^{\prime}: 8,7\right]$ cyclooct $[b]$ indole-6-carboxylic acid (11a). A similar series of compounds were derived from 6a-d to yield 11a-d.

\subsection{Mechanism for the formation of product $\mathbf{1 1}$}

As shown in scheme 5, the isatin is converted by the action of sodium hydroxide into the salts of isotoic acid $\mathbf{I}$. Isotoic acid $\mathbf{I}$ condenses with 1-oxo-1,2, 3,4,5,6-hexahydrocyclooct $[b]$ indole $\mathbf{6}$ releases water, forming the salt II. The salt II undergoes tautomerization followed by prototropic shift gave the intermediate III. The latter III undergo aromatization with the release of water gave salts of 7,8,9,10tetrahydroquinolino $\left[2^{\prime}, 3^{\prime}: 8,7\right]$ cyclooct $[b]$ indole-6-car- 


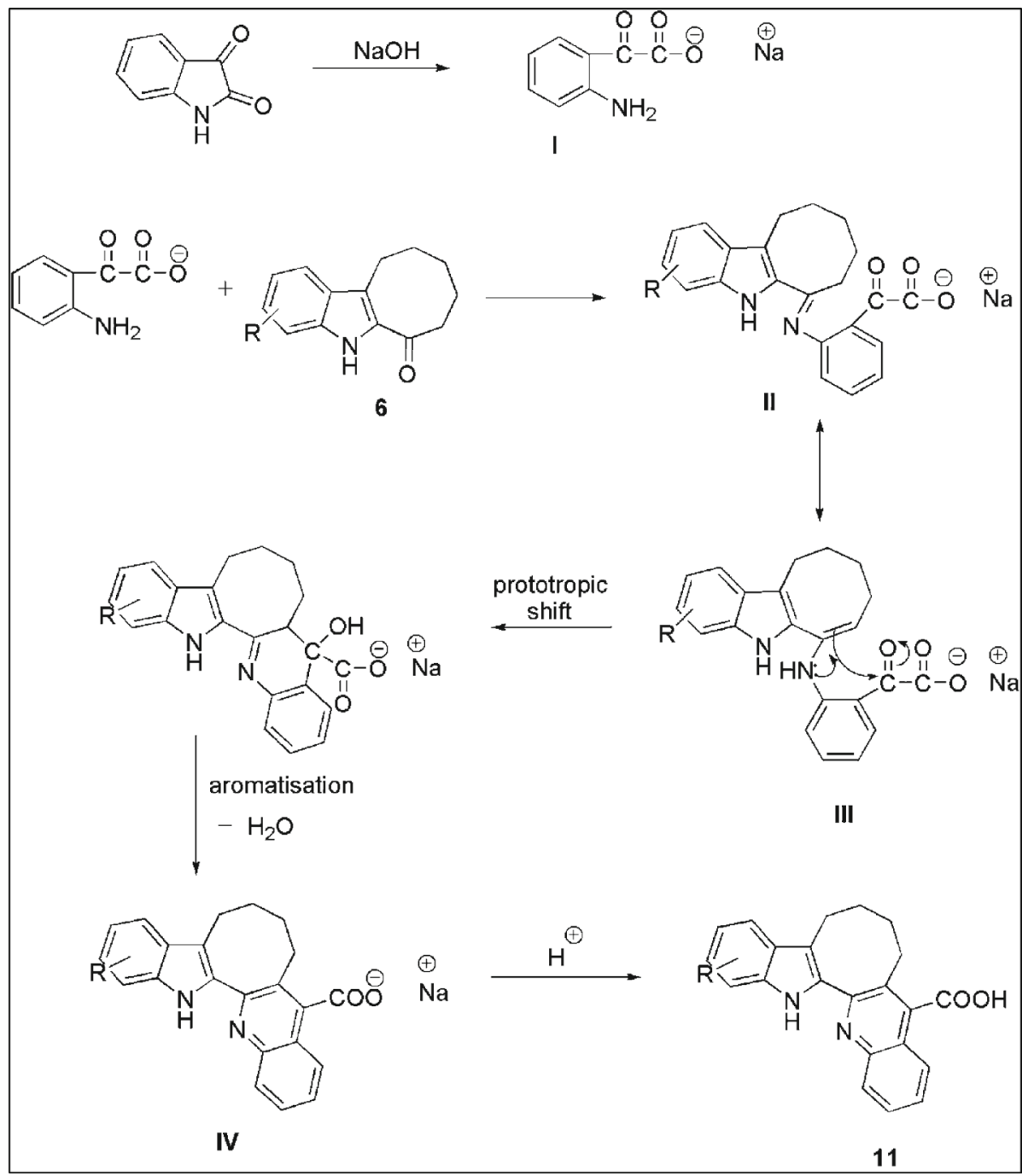

Scheme 5. Mechanism for the formation of product 11.

Table 1. Comparison of the yield of the product $\mathbf{1 1}$.

\begin{tabular}{|c|c|c|c|c|c|}
\hline \multirow[b]{2}{*}{$\begin{array}{l}\text { Compounds } \\
3,4,5\end{array}$} & \multirow[b]{2}{*}{$\mathbf{R}^{1}$} & \multirow[b]{2}{*}{$\mathbf{R}^{2}$} & \multirow[b]{2}{*}{$\mathbf{R}^{3}$} & \multicolumn{2}{|c|}{ Product 11} \\
\hline & & & & $\begin{array}{l}\text { Yield using } \\
\mathrm{NaOH}(\%)\end{array}$ & $\begin{array}{l}\text { Yield using } \\
\mathrm{KOH}(\%)\end{array}$ \\
\hline $\mathbf{a}$ & $\mathrm{H}$ & $\mathrm{H}$ & $\mathrm{CH}_{3}$ & 37 & 85 \\
\hline b & $\mathrm{CH}_{3}$ & $\mathrm{H}$ & $\mathrm{H}$ & 32 & 80 \\
\hline c & $\mathrm{Cl}$ & $\mathrm{H}$ & $\mathrm{H}$ & 27 & 82 \\
\hline d & $\mathrm{H}$ & $\mathrm{H}$ & $\mathrm{H}$ & 41 & 78 \\
\hline
\end{tabular}

boxylic acid IV, the treatment of which with acid gives the required compound $\mathbf{1 1 .} .^{22}$

\section{Conclusion}

From the literature survey and to the best of our knowledge, first time we report here the rapid synthesis of 6-chloro-7,8,9,10-tetrahydroquinolino[ $\left[2^{\prime}, 3^{\prime}: 8,7\right]$ cyclooct $[b]$ indole, 6-chloro-7,8,9,10-tetrahydro(pyrazino[2" $\left.2^{\prime \prime} 3^{\prime \prime}-\mathrm{e}^{\prime}\right]$ pyrido)-[2', $\left.3^{\prime}: 8,7\right]$ cyclooct $[b]$ indole and 6-chloro-7,8,9,10-tetrahydroquinolino [2' $\left.2^{\prime}: 8,7\right]$ cyclooct $[b]$ indole-6-carboxylic acids. The methodology of the synthesis of a variety of novel bioactive cyclooct $[b]$ indole incorporating quinoline and pyrazino pyrido moiety under $\mathrm{POCl}_{3}$ conditions using 1-oxo$1,2,3,4,5,6$-hexahydrocyclooct $[b]$ indoles with anthranillic acid and 3-amino pyrazine acid and isatin has been developed and their structural features were identified. The present methodology gives significant advantages such as simple procedure, easy work-up, clean reaction profile and excellent percentage of yields.

\section{Supplementary information}

The ${ }^{1} \mathrm{H}$ and ${ }^{13} \mathrm{C}$ NMR spectra of all new compounds are included in the supplementary information (see www. ias.ac.in/chemsci). 


\section{Acknowledgements}

We thank the Indian Institute of Technology (IIT) Madras, Chennai, and Indian Institute of Chemical Technology (IICT) Hyderabad, India for their support in spectral studies.

\section{References}

1. Humphrey G R and Kuethe J T 2006 Chem. Rev. 106 2875

2. Keawpradub N, Eno-Amooquaye E, Burke P J and Houghton P 1999 Planta Med. 65311

3. Mayerl F and Hesse M 1978 Helv. Chim. Acta 61 337

4. Keawpradub N, Kirby G C, Steele J C P and Houghton P J 1999 Planta Med. 65690

5. Wright C W, Allen D, Cai Y, Phillipson J D, Said J M, Kirby G C and Warhurst D C 1992 Phytother. Res. 6121

6. Edwankar C R, Edwankar R V, Deschamps J R and Cook J M 2012 Angew. Chem. Int. Ed. 51 11762-11765.

7. Jampilek J, Dolezal M, Kunes J, Buchta V and Kralova, K 2005 Med. Chem. 1591

8. Roth H J, Fenner H. 2000 In Arzneistoffe, 3rd ed. In Deutscher Apotheker; Verlag: Stuttgart; pp 51114

9. Musiol R, Jampilek J, Buchta V, Silva L, Niedbala, H, Podeszwa B, Palka A, Majerz-Maniecka K, Oleksyn B and Polanski J 2006 Bioorg. Med. Chem. 143592

10. Harris C R and Thorarensen A 2004 Curr. Med. Chem. 112213
11. Vangapandu S, Jain M, Jain R, Kaur S and Singh P P 2004 Bioorg. Med. Chem. 122501

12. Andries K, Verhasselt P, Guillemont J, Gohlmann H W, Neefs J M, Winkler H, Van Gestel J, Timmerman P, Zhu M, Lee E, Williams P, de Chaffoy D, Huitric E, Hoffner S, Cambau E, Truffot-Pernot C, Lounis N and Jarlier V 2005 Science $\mathbf{3 0 7} 223$

13. Vinsova J, Imramovsky A, Jampilek J, Monreal-Ferriz J and Dolezal M 2008 Anti-infective Agents Med. Chem. 712

14. Sissi C and Palumbo M 2003 Curr. Med. Chem. Anti-Canc. Agents 3439

15. Mekouar K, Mouscadet J F, Desmaele D, Subra F, Leh, H, Savoure D, Auclair C and d'Angelo J 1998 J. Med. Chem. 412846

16. Polanski J, Zouhiri F, Jeanson L, Desmaele D, d'Angelo J, Mouscadet J, Gieleciak R, Gasteiger J and Bret M L 2002 J. Med. Chem. 454647

17. Polanski J, Niedbala H, Musiol R, Tabak D, Podeszwa B, Gieleciak R, Bak A, Palka A and Magdziarz T 2004 Acta Poloniae Pharm. Drug Res. 613

18. Pommier Y, Johnson A A and Marchand C 2005 Nat. Rev. Drug. Discov. 4236

19. Zouhiri F, Danet M, Bernard C, Normand-Bayle M, Mouscadet J F, Leh H, Thomas C M, Mbemba G, d'Angelo J and Desmaele D 2005 Tetrahedron Lett. 46 2201

20. Prabakaran K and Prasad K J R 2010 Syn. Comm. 40 3528

21. Pfitzinger W 1886 J. Prakt. Chem. 33100

22. Prabakaran K 2009 Synthetic Studies on Heteroannulated Carbazoles, Ph.D. Thesis, Bharathiar University, Coimbatore, Tamil Nadu, India 